\title{
Diagrammatic method for investigating universal behavior of impurity systems
}

\author{
Kurt Fischer \\ Max-Planck-Institut für Physik komplexer Systeme, Bayreuther Strasse 40, 01187 Dresden, Germany
}

(November 18th)

\begin{abstract}
The universal behavior of magnetic impurities in a metal is proved with the help of skeleton diagrams. The energy scales are derived from the structure of the skeleton diagrams. A minimal set of skeleton diagrams is sorted out that scales exactly. For example, the non-crossing approximation for the Anderson impurity model can describe the crossover phenomenon. The universal Wilsonnumber is calculated within the non-crossing approximation. The method allows for an assessment of various approximations for impurity Hamiltonians.
\end{abstract}

\section{INTRODUCTION}

Magnetic impurities in metals show universal behavior at low energiest. The Hamiltonian of such systems consists of at least one conduction band of width $D$ to which the impurity couples2. At temperatures $k_{B} T \ll D$, the scaled observable is independent of details of the host system such as its band structure.

A long standing question in the physics of such systems is: How can the observed universal behavior directly be established from the original model Hamiltonian such as the Anderson-impurity model2, together with a reasonable accurate description of observables? Hitherto the original Hamiltonian has been replaced by another one which is more accessible:

(a) Within the Bethe ansatz the impurity part of the system's thermodynamics can be derived. However, the original model has to be replaced by one with linear dispersion. The spectrum of eigenvalyes is cut off at $D^{\prime}$ which is in general not identical with the band width $D$ of the metallic host in the original model 3 . A. A relation between $D$ and $D^{\prime}$ has so far been established only for the Anderson impurity model 5 .

(b) Within the numerical renormalization group6, the impurity is coupled to a half-infinite chain with hopping matrix elements vanishing as $\Lambda^{-n}, n=1,2, \ldots, \Lambda>1$. In the limit of $\Lambda=1$ one would recover the original model. However, the numerical results have to be extrapglated to that limit because the length of the chain to be diagonalized numerically would eventually become too large 6 .

Hence both methods do not prove that the observables of the original model behave universally. On the other hand, there are the diagrammatic approaches to the impurity problem . They allow us to construct approximations for all observables of the original model. The Dyson equation

$$
R^{-1}(z)=R_{0}^{-1}(z)-\Sigma(z)
$$

is invariant under a certain rescaling of the propagators $R$ and $R_{0}$, self-energies $\Sigma$, and coupling constants. With the help of the diagrammatic renormalization group, perturbative results for the propagators, self-energies, and vertex parts are then fitted to the Dyson equation. In that way, scaling lays for the propagators result, from which the universal behavior in the perturbative high-energy regime of the model 8 follows. This method amounts to summing a certain subclass of diagrams, with naked propagators. However, the procedure breaks down when perturbation theory fails.

For the nonperturbative region dressed propagators are necessary. This requires the use of skeleton diagrams, otherwise the definition of a self-energy itself would become ambiguous. However, the scaling of the dressed propagators is unknown, precisely because beforehand the Dyson equation would have to be solved.

In this paper, this difficulty is overcome by utilizing the variational principle of Luttinger and Ward 9 , Kuramotd 10 , and Baym 11, by which any observable can be expressed in terms of skeleton diagrams. This is exemplified at the Anderson-impurity Hamiltonian.

It turns out that the skeleton diagrams of the second order are already sufficient to calculate the exact energy scales. This proves universal behavior for this model. The second-order skeleton diagrams are therefore the minimal class of diagrams which have to be summed, in order to describe the crossover. 


\section{HAMILTONIAN AND DIAGRAM TECHNIQUE}

As the standard model describing magnetic impurities in metals, the Anderson-impurity Hamiltonian is considered, with a half-filled conduction band of constant density of states $\rho$ and infinite Coulomb repulsion at the impurity site:

$$
\begin{aligned}
H & =H_{c}+H_{f}+H_{1}, \\
H_{c} & =\sum_{\left|\epsilon_{p}\right| \leq D, m}, \epsilon_{p} c_{p m}^{+} c_{p m} \\
H_{f} & =\epsilon_{f} \sum_{m} f_{m}^{+} f_{m} \\
H_{1} & =\frac{V}{\sqrt{N}} \sum_{p, m},\left(c_{p m}^{+} b^{+} f_{m}+\text { H.c. }\right) .
\end{aligned}
$$

$c_{p m}^{+}$creates a conduction electron with internal quantum number $m=1 \ldots N$, momentum $p$, and energy $\epsilon_{p}$ which is cut off at $\pm D .|m\rangle=f_{m}^{+}|\mathrm{vac}\rangle$ denotes a magnetic configuration of the impurity and $|0\rangle=b^{+} \mid$vac $\rangle$the non-magnetic one, their energy-difference being $\epsilon_{f} . f_{m}^{+}$is a fermionic operator and $b^{+}$the Coleman boson 12 . $F_{m}^{+}=f_{m}^{+} b$ creates an electron at the impurity site. Double occupancy at the impurity site is suppressed by imposing the constraint $n_{b}+n_{f}=1$. The impurity hybridization with the conduction band is proportional to $V$. The Boltzmann constant is set to unity so that temperature is measured in units of energy.

In order to simplify the subsequent derivations, the conduction band of model (1) is assumed to have a constant density of states $\rho$ with a sharp cutoff at energies $\pm D$, as the other approaches to scaling do 2,3 . Universal behavior should not depend on this assumptionB. In Sec. VII it will be shown that the energy scales of the system indeed do not change as long as the density of states is finite at the Fermi energy, and is sufficient structureless to have only one energy scale $D$.

The principal object of concern is the resolvent $R_{f}(z)=\langle Q(z)\rangle_{c}$ where $Q$ is defined as

$$
Q(z)=1 /\left(z-L_{c}-H_{f}-H_{1}\right) \text {. }
$$

Here the superoperator $L_{c}$ acts on an operator $X$ of the Hilbert space as $L_{c} X=\left[H_{c}, X\right]$, and \langle\rangle$_{c}$ indicates the thermodynamic average with respect to $H_{c}$. The propagators for the occupied and unoccupied ionic configurations are

$$
\begin{aligned}
R_{m}(z) & :=\left\langle m\left|R_{f}(z)\right| m\right\rangle \\
R_{0}(z) & :=\left\langle 0\left|R_{f}(z)\right| 0\right\rangle .
\end{aligned}
$$

With the help of the identity 13

$$
e^{-\beta H}=e^{-\beta\left(L_{c}+H_{f}+H_{1}\right)} e^{-\beta H_{c}}
$$

the impurity part $Z_{f}$ of the partition function can then be represented as a line integral, the path of integration encircling all poles of the integrand:

$$
Z_{f}:=\frac{\operatorname{Tr}_{f} \operatorname{Tr}_{c} e^{-\beta H}}{\operatorname{Tr}_{c} e^{-\beta H_{c}}}=\operatorname{Tr}_{f} \oint \frac{d z}{2 \pi i} e^{-\beta z} R_{f}(z)
$$

The well-known diagrammatic technique 14 follows if $Q$ is expanded in a geometric series in $V$, then $L_{c}$ acting on the conduction-electron operators in $H_{1}$ is evaluated to give the energy denominators

$$
L_{c}\left(c_{p m}^{+} c_{q n} \ldots\right)=\left(\epsilon_{p}-\epsilon_{q}+\cdots\right)\left(c_{p m}^{+} c_{q n} \cdots\right)
$$

and finally Wick's theorem applied to evaluate the thermodynamic average with respect to $H_{c}$. This can be casted in a diagrammatic language. The naked propagators and vertices are shown in Fig. 1. Because the impurity site can alternatively be empty or singly occupied, every diagram has a spine of alternating $b$ and $f$ propagators. Consequently, all diagrams where a conduction-electron propagator would have a self-energy are excludedi4, because they do not fulfill the constraint $n_{b}+n_{f}=1$. Alternative approaches where this constraint is not exactly enforced will be discussed in Sec. VIII.

Within the variational principle 11 , a functional $\Upsilon$ of the dressed one-particle propagators is defined. Because the conduction-electron propagators carry no self-energy, the variational principle has been adapted to the present 
diagrammatic technique 10 such that $\Upsilon$ becomes a functional of the dressed one-particle impurity-propagators only. At the saddle point with respegt to variations of the $R_{0, m}$, the functional $\Upsilon$ equals $Z_{f}$, and the Dyson equation holds as a self-consistency equation 10

$$
R_{f}(z)^{-1}=z-H_{f}-\Sigma_{f}(z)
$$

For the impurity-part of the partition function, the functional is given by

$$
\begin{aligned}
\Upsilon= & \beta \operatorname{Tr}_{f} \oint \frac{d z}{2 \pi i} e^{-\beta z}\left\{\sum_{n}\left(1-\frac{1}{n}\right) \Sigma_{f}^{(n)}(z) R_{f}(z)\right. \\
& \left.+\ln \left[z-H_{f}-\sum_{n} \Sigma_{f}^{(n)}(z)\right]\right\} .
\end{aligned}
$$

Here $\Sigma_{f}^{(n)}$ denotes all $n$th order self-energy diagrams of $R_{f}$, expressed in terms of skeleton diagrams. Skeleton diagrams are diagrams where all self-energy insertions have been removed. Hence a skeleton diagram becomes a functional of the dressed propagators.

The variational principle can be interpreted in the following fashion: If $\Upsilon$ depends on some parameter $\lambda$ such 2 s the hybridization then, at the saddle point, it depends on $\lambda$ only explicitly, and not implicitly via the propagator 10 :

$$
\frac{d \Upsilon}{d \lambda}=\frac{\partial \Upsilon}{\partial \lambda}+\frac{\delta \Upsilon}{\delta R_{0, m}} \frac{\partial R_{0, m}}{\partial \lambda}=\frac{\partial \Upsilon}{\partial \lambda} .
$$

Explicitly, from Eq. (5) it follows at the saddle point that

$$
\begin{aligned}
\frac{d Z_{f}}{d \lambda}= & -\frac{d}{d \lambda} \beta \operatorname{Tr}_{f} \oint \frac{d z}{2 \pi i} e^{-\beta z} \sum_{n} \frac{1}{n} \Sigma_{f}^{(n)}(z) R_{f}(z) \\
& -\frac{d}{d \lambda} \beta \operatorname{Tr}_{f} \oint \frac{d z}{2 \pi i} e^{-\beta z} R_{f}(z) H_{f}
\end{aligned}
$$

where the $\lambda$ dependence of $R_{f}$ can be discarded.

In order to study the dependence of $Z_{f}$ on a parameter $\lambda$, it therefore suffices to consider all skeleton diagrams of the type $\Sigma_{0, m}^{(n)} R_{0, m}$. A skeleton diagram for $\Sigma_{0}^{(2)} R_{0}$, i.e. of second order, is shown in Fig. 2 .

Approximations fulfilling the variational principle can be generated by using a subclass, a so-called family of skeleton diagrams 15 , which contains with each skeleton diagram for $\Sigma_{0, m}^{(n)} R_{0, m}$ all others with cyclic permuted vertices as well. For example, all skeleton diagrams of a given order $\Sigma_{0, m}^{(n)} R_{0, m}$ form such a family.

For instance, if only skeleton diagrams of second order are kept in Eq. (5), this amounts to summing all diagrams with bare propagators and noncrossing conduction-electron lines and is called the NCA 10 . The self-energies of the $\mathrm{NCA}, \Sigma_{m}^{(2)}(z)=\Sigma_{m}^{(2)}\left[R_{0}(z)\right]$ and $\Sigma_{0}^{(2)}(z)=\Sigma_{0}^{(2)}\left[R_{1}(z), \ldots, R_{n}(z)\right]$, are then $(f$ denotes the Fermi function)

$$
\begin{aligned}
& \Sigma_{m}^{(2)}(z)=\frac{V^{2} \rho}{N} \int_{-D}^{D} f(\epsilon) R_{0}(z+\epsilon) d \epsilon, \\
& \Sigma_{0}^{(2)}(z)=\frac{V^{2} \rho}{N} \sum_{m=1}^{N} \int_{-D}^{D} f(\epsilon) R_{m}(z+\epsilon) d \epsilon .
\end{aligned}
$$

The functional $\Upsilon^{(2)}$ has then the form

$$
\begin{aligned}
\Upsilon^{(2)}= & \oint \frac{\beta d z}{2 \pi i} e^{-\beta z}\left\{\frac{1}{2} \Sigma_{0}^{(2)}(z) R_{0}(z)+\frac{1}{2} \sum_{m} \Sigma_{m}^{(2)}(z) R_{m}(z)\right. \\
& \left.+\ln \left[z-\Sigma_{0}^{(2)}(z)\right]+\sum_{m} \ln \left[z-\epsilon_{f}-\Sigma_{m}^{(2)}(z)\right]\right\} .
\end{aligned}
$$

To construct a variational principle for other observables the Hamiltonian has to be coupled to external fields suitably chosen 10. This will be discussed in Sec. D. 


\section{ENERGY SCALES}

\section{A. First scaling equation}

$\Upsilon$ as defined in Eq. (5) depends explicitly on $\epsilon_{f}$ oply via the term $H_{f}=\epsilon_{f} \sum_{m} f_{m}^{+} f_{m}$. Consequently, one has for the impurity part $F_{f}=-T \ln Z_{f}$ of the free energy 4 :

$$
Z_{f} \epsilon_{f} \partial_{\epsilon_{f}} F_{f}=\operatorname{Tr}_{f} H_{f} \oint \frac{d z}{2 \pi i} e^{-\beta z} R_{f}(z) .
$$

$\Upsilon$ depends explicitly on $V$ via 10 the prefactor $V^{2 n}$ of the $2 n$th order self-energy $\Sigma_{f}^{(2 n)}$ :

$$
Z_{f} \rho V^{2} \partial_{\rho V^{2}} F_{f}=\frac{1}{2} \operatorname{Tr}_{f} \oint \frac{d z}{2 \pi i} e^{-\beta z} \Sigma_{f}(z) R_{f}(z) .
$$

To determine the $T$ dependence, the internal integration variables $z$ and $\epsilon$ as in Eqs. (5), and (6) are replaced by $T z$ and $T \epsilon$, respectively. The variational principle remains unaffected. $\Upsilon$ depends now explicitly on $T$ via the prefactor $T^{n}$ of a skeleton diagram of $2 n$th order, the term $T z$ in the logarithm, and the integration boundaries as in Eq. (6) change to $\pm D / T$. Therefore

$$
\begin{gathered}
Z_{f}\left(F_{f}-T \frac{\partial}{\partial T} F_{f}\right)=\frac{1}{2} \operatorname{Tr}_{f} \oint \frac{d z}{2 \pi i} e^{-\beta z} \Sigma_{f}(z) R_{f}(z) \\
+Z_{f} D \frac{\partial}{\partial D} F_{f}+\operatorname{Tr}_{f} H_{f} \oint \frac{d z}{2 \pi i} e^{-\beta z} R_{f}(z) .
\end{gathered}
$$

Inserting Eq. (7) and Eq. (8) into Eq. (9) yields

$$
F_{f}=\left(T \frac{\partial}{\partial T}+\rho V^{2} \frac{\partial}{\partial \rho V^{2}}+\epsilon_{f} \frac{\partial}{\partial \epsilon_{f}}+D \frac{\partial}{\partial D}\right) F_{f} .
$$

This equation expresses the scaling of $F_{f}$ with respect to the energy scales $D, \epsilon_{f}$, and $\rho V^{2}$ :

$$
F\left(T, \rho, V, \epsilon_{f}, D\right)=T f\left(\frac{T}{\rho V^{2}}, \frac{T}{\epsilon_{f}}, \frac{T}{D}\right)
$$

This is henceforth called the first scaling equation.

\section{B. Second scaling equation}

The central issue of this work is to prove and describe the universal behavior of impurity systems. To show the universal behavior for $F_{f}$, the functional $\Upsilon$ is examined for large but finite $D$, that is, when $D$ becomes larger than all other energy scales of the system, the so-called universal limit 3 .

$\Upsilon$ depends explicitly on the cutoff $D$ only via the integration boundaries $\pm D$ of the integration over the conductionelectron energies, as can be seen in Eq. (6) for the second-order skeleton diagrams.

At first only those second-order self-energies are kept in $\Upsilon$. With the help of the spectral densities $\rho_{0, m}$ of $R_{0, m}$ one has

$$
\begin{aligned}
D \frac{\partial}{\partial D} F_{f}^{(2)}= & \frac{V^{2} \rho}{N Z_{f}} \sum_{\substack{m=1 \\
\mu= \pm 1}}^{N} \int e^{-\beta \omega} d \omega D f(\mu D)\left[\rho_{m}(\omega)\right. \\
& \left.\times \Re R_{0}(\omega+\mu D)+\rho_{0}(\omega) \Re R_{m}(\omega+\mu D)\right]
\end{aligned}
$$

where $\Re R_{0, m}$ denotes the real part of $R_{0, m}$. In the universal limit, in particular $T / D \rightarrow 0$, so that only the terms $\propto$ $f(-D) \approx 1$ survive. Furthermore, in this limit the weighted spectral densities $e^{-\beta \omega} \rho_{0, m}(\omega) / Z_{f}$ contribute significantly only for frequencies less then the impurity part of the ground-state energy $E_{0}<0$, because $e^{\beta E_{0}} Z_{f}$ tends to 1 for low temperatures. From perturbation theory it follow 10 that $e^{-\beta \omega} \rho_{f}(\omega) / Z_{f}$ vanishes asymptotically as $1 / \omega^{2}$ for large, negative $\omega$. Hence it contributes significantly to the integral in Eq. (11) only for 


$$
-\sqrt{D} \lesssim \omega \lesssim E_{0}
$$

In this frequency interval, $\Re R_{f}(\omega-D)$ can be replaced by its bare counterpart $1 /\left(\omega-H_{f}-D\right) \approx(-1) / D$ :

$$
D \frac{\partial}{\partial D} F_{f}^{(2)}=\frac{-V^{2} \rho}{N Z_{f}} \sum_{m=1}^{N} \int e^{-\beta \omega} d \omega\left[\rho_{m}(\omega)+\rho_{0}(\omega)\right] .
$$

Together with Eq. (7), the following scaling relation is obtained:

$$
D \frac{\partial}{\partial D} F_{f}^{(2)}=\rho V^{2}(1-1 / N) \frac{\partial}{\partial \epsilon_{f}} F_{f}^{(2)}-\rho V^{2} .
$$

This is called henceforth the second scaling equation. Next it is shown that in the universal limit all families of higher order skeleton diagrams of $\Upsilon$ are irrelevant, by which is meant here that their contribution to the logarithmic derivative in Eq. (12) vanishes as $O(1 / D)$. For the proof see Appendix $\mathrm{A}$, the families of skeleton diagrams being needed to enforce the variational principle.

The result can be made plausible by casting it into the language of diagrams, as in Fig. 2. Differentiating logarithmically with respect to $D$ means removing one curved conduction-electron line and replacing the internal propagator by its value at the cutoff $\propto 1 / D$. Therefore this diagram contributes $\propto D / D$ to the logarithmic derivative.

For a diagram of higher order than two such as in Fig. 3, there lie under each conduction-electron line at least three propagators, because otherwise this diagram would have a self-energy insertion. Hence its contribution to the logarithmic derivative is $\propto D / D^{3}$ and can be neglected for large $D$.

Equation (12) therefore holds as well for the exact $F_{f}$. It describes the Haldane scaling17 for the difference $F_{f}-E_{0}$, that is, it depends on $D$ and $\epsilon_{f}$ only via

$$
\begin{gathered}
\left(F_{f}-E_{0}\right)\left(T, V, \rho, \epsilon_{f}, D\right)=\left(F_{f}-E_{0}\right)\left(T, V, \rho, \epsilon_{f}^{*}\right), \\
\epsilon_{f}^{*}=\epsilon_{f}+(1-1 / N) \rho V^{2} \ln D /\left(\rho V^{2}\right) .
\end{gathered}
$$

By inserting this into Eq. (10), in the magnetic limit $-\epsilon_{f} \gg \rho V^{2}$ the scaling law

$$
F_{f}-E_{0}=T g\left(T, V, \epsilon_{f}, D, \rho\right)=T g\left(\frac{T}{\Gamma}, \frac{T}{T_{K}}\right)
$$

follows, with the quantity $\Gamma=\pi \rho V^{2} / N$ and (up to a numerical factor) the Kondo temperaturel

$$
T_{K}=D \sqrt[N]{\rho V^{2} / D} \exp \left[\epsilon_{f} / \rho V^{2}\right] .
$$

This proves the universal behavior for the free energy of the Anderson Hamiltonian as well as that the energy scales $\Gamma$ and $T_{K}$ are the exact ones. In particular, it has been shown that the NCA preserves the exact energy scales of the system. Because the NCA is exact up to orders $V^{4}$ and $1 / N$, an inclusion of families of skeleton diagrams of higher order in Eq. (13) will slightly change $g$ but not the energy scale and consequently will not alter the approximation qualitatively. Such an extension of the NCA has been performed 18 and within the errors of the numerical calculation the scaling law (13) as well as a slight change in the respective function $g$ have been verified.

There holds an analogy to the usual diagrammatic renormalization group, as described in Sec. [1. It turns out that from a certain order in perturbation theory on, the energy scales obtained by that method do not change any more in the universal limit, while, for an observable the form of the respective function $g$ can still change. However, the point is that this scheme can only be used for high temperatures $T \gg T_{K}$, below which perturbation theory breaks down. Hence a necessary condition for a diagrammatic technique to describe the universal behavior of impurity systems is that it includes families of skeleton diagrams including those of second order. This result can straightforwardly be extended to the case of finite magnetic fields.

\section{SCALING WITH MAGNETIC FIELD}

The influence of a magnetic field $h$ on the host metal is of order $h / D \propto 1 / D$, and can be neglected. Hence, to the Hamiltonian of Eq. (11) only 


$$
H_{f} \longrightarrow H_{f}+g \mu_{B} h \sum_{m} m f_{m}^{+} f_{m}
$$

is added, where $g$ denotes the $g$ factor of the $f$ electron and $\mu_{B}$ is the Bohr magneton. The functional $\Upsilon$ will explicitly depend on $h$ only via the new term in $H_{f}$. Consequently the first scaling equation (10) changes to

$$
\begin{aligned}
F= & T \frac{\partial}{\partial T} F+\rho V^{2} \frac{\partial}{\partial \rho V^{2}} F+\epsilon_{f} \frac{\partial}{\partial \epsilon_{f}} F \\
& +g \mu_{B} h \frac{\partial}{\partial g \mu_{B} h} F+D \frac{\partial}{\partial D} F .
\end{aligned}
$$

The second scaling equation (12) remains unaffected. The scaling law for the magnetization $M(h)=-(\partial / \partial h) F_{f}$ therefore reads

$$
M\left(T, V, \epsilon_{f}, D, \rho, h\right)=M\left(\frac{T}{\Gamma}, \frac{T}{T_{K}}, \frac{h}{\Gamma}, \frac{h}{T_{K}}\right) .
$$

\section{PROOF OF UNIVERSALITY}

To obtain the scaling of any observable, the Hamiltonian $H$ has to be coupled to suitable external fields. However, only the skeleton diagram of Fig. 2 is relevant for large $D$. Analogous scaling equations such as Eqs. (10) and (13) can thus be derived for any other observalple, hence proving universality for the Anderson model.

As an example, take the $f$ propagator 14 as a function of imaginary time $\tau$,

$$
G_{f m}(\tau)=-\left\langle T_{\tau} F_{m}(\tau) F_{m}^{+}(0)\right\rangle .
$$

Here $T_{\tau}$ is the time-ordering operator, and \langle\rangle denotes the thermodynamic average with respect to $H$. With the help of $Q(z)$ one can reproduce the well-known integral representation for $G_{f m}$ which coincides for fermionic Matsubara frequencies with the Fourier transform of $G_{f m}(\tau)$ e

$$
G_{f m}(\omega)=\oint e^{-\beta z} \frac{d z}{2 \pi i}\langle\langle 0|Q(z)| 0\rangle\langle m|Q(z+\omega)| m\rangle\rangle_{c} .
$$

To obtain a variational functional for $G_{f m}, H$ is coupled to an auxiliary fermionic field $\Psi$ :

$$
H+\omega \Psi^{+} \Psi+V \sqrt{\rho} \lambda\left(\Psi^{+} b^{+} f_{m}+H . c .\right) .
$$

The $f$ propagator is obtained from all diagrams of second order in $\lambda$ that is, after removing the $\Psi$ propagator 1 . A functional $\Upsilon_{\Psi}$ can be constructed analogously to Eq. (5), to give, at the saddle point,

$$
Z_{\Psi}:=\frac{\operatorname{Tr}_{f} \operatorname{Tr}_{c} \operatorname{Tr}_{\Psi} e^{-\beta H}}{\operatorname{Tr}_{c} \operatorname{Tr}_{\Psi} e^{-\beta\left(H_{c}+H_{\Psi}\right)}}
$$

$G_{f}$ follows as

$$
\left.\frac{\partial}{\partial \lambda^{2}}\right|_{\lambda=0} F_{\Psi}=\rho V^{2} f(\omega) G_{f m}(\omega)
$$

The scaling equations for $F_{\Psi}$, and via Eq. (19) for $G_{f}$, can be established in the same manner as for $F_{f}$. In particular, Eq. (10) now reads

$$
\left(T \frac{\partial}{\partial T}+\omega \frac{\partial}{\partial \omega}+\epsilon_{f} \frac{\partial}{\partial \epsilon_{f}}+\rho V^{2} \frac{\partial}{\partial \rho V^{2}}+D \frac{\partial}{\partial D}+1\right) G_{f m}=0
$$

In the universal limit of large $D$ again only the skeleton diagrams of second order are relevant, and the analog to Eq. (12) holds for $G_{f}$, too:

$$
D \frac{\partial}{\partial D} G_{f m}=\rho V^{2}(1-1 / N) \frac{\partial}{\partial \epsilon_{f}} G_{f m} .
$$

In the magnetic limit $-\epsilon_{f} \gg \Gamma$ where $\Gamma \gg T_{K}$ it follows that the Abrikosov-Suhl resonance and the impurity part of the resistivity computed within the NCA scale with the exact Kondo temperature. This was numerically observed in Ref. 20. There, the energy scale was determined as the maximum value of the spectral density of $G_{f m}(\omega)$ in the magnetic limit of the Anderson model, and assumed to be proportional to $T_{K}$. Here this has been proved. 


\section{DESCRIPTION OF CROSSOVER: WILSON NUMBER}

Within this scaling method the crossover phenomenon 6 can now be described entirely within a diagrammatic approach. Even for the skeleton diagrams of second order an analytical solution of the self-consistency equations does not seem possible for finite temperature. However, for zero temperature the well-known expressions for the ground-state energy of the NCA10,21 for the Anderson model can be evaluated analytically (see Appendix B) in the magnetic limit. In this limit, it follows from Eq. (17) that for zero temperature the scaling law

$$
M\left(V, \epsilon_{f}, D, \rho, h\right)=M\left(\frac{h}{T_{K}}\right)
$$

holds, which holds as well for the NCA, as shown above. For low magnetic fields, the law-field energy scale $T_{L}$ can be fixed unambiguously by the static magnetic susceptibility at vanishing magnetic field,

$$
\chi(0)=\frac{1}{3} \mu_{j}^{2} / T_{L} .
$$

With the help of the analytical NCA results of Appendix B, an analytical expression for $T_{L}$ is obtained:

$$
T_{L}^{\mathrm{NCA}}=T_{K} / \Gamma(1-1 / N),
$$

with $\Gamma$ being the gampa function. The result coincides up to order $1 / N$ with the result $T_{L}=T_{K} \Gamma(1+1 / N)$ which is believed to be exact 3 .

For high magnetic fields $g \mu_{B} h \gg T_{K}$, the NCA reproduces the perturbation theory up to $V^{4}$. With the definitions $N=2 j+1$ and $J=V^{2} /\left|\epsilon_{f}\right|$, for the impurity part of the magnetization the expansion

$$
\begin{aligned}
\frac{M(h)}{j g \mu_{B}}= & 1-\frac{\rho J}{N}+\frac{(\rho J)^{2}}{N} \ln \frac{g \mu_{B} h \sqrt[N]{e}}{D \sqrt[N]{\epsilon_{f} / D}} \\
& +\frac{(\rho J)^{2}}{N}\left[\frac{2}{N(N-1)} \sum_{m=1}^{N-1} m \ln (m)\right]+O\left(J^{3}\right)
\end{aligned}
$$

is obtained. The exact scaling (21) of the NCA renders it possible to reexpress in the perturbation expansion $J$ in terms of $h / T_{K}$. The high-field energy scale $T_{H}$ is fixed unambiguously 2 by requiring that terms $\propto 1 / \ln ^{2}\left[g \mu_{B} h / T_{H}\right]$ to be absent in the resulting asymptotic expansion. One arrives at the well-known asymptotic renormalization-group result for the magnetization in high magnetic fields

$$
\begin{aligned}
\frac{M(h)}{j g \mu_{B}}= & 1-\frac{1}{N} \frac{1}{\ln \frac{g \mu_{B} h}{T_{H}}}-\frac{1}{N^{2}} \frac{\ln \ln \frac{g \mu_{B} h}{T_{H}}}{\ln ^{2} \frac{g \mu_{B} h}{T_{H}}} \\
& +O\left(\frac{\ln \ln \frac{g \mu_{B} h}{T_{H}}}{\ln ^{3} \frac{g \mu_{B} h}{T_{H}}}\right)
\end{aligned}
$$

and $T_{H}$ is given by

$$
T_{H}=T_{K} \exp \left[-1 / N-\frac{2}{N(N-1)} \sum_{m=1}^{N-1} m \ln (m)\right] .
$$

The Wilson ratio $W=T_{H} / T_{L}$ characterizes the crossover from the high-field region where the impurity reacts as an asymptotically free magnetic moment, to the low-energy region where the impurity is screened. Hence the Wilson ratio coincides with the exact result up to order $1 / N$ :

$$
\begin{aligned}
\frac{W^{\mathrm{NCA}}}{W} & =\frac{1}{\Gamma(1-1 / N) \Gamma(1+1 / N)} \\
& =1+O\left(1 / N^{2}\right) .
\end{aligned}
$$

By a Schrieffer-Wolff transformation 1 , this result can be extended to the Coqblin-Schrieffer model (see Appendix Q). 


\section{HOW MUCH DOES SCALING DEPEND ON BAND STRUCTURE?}

The universal behavior of impurity models like the Anderson model should not depend on details of the host's density of states as long as its band has a finite density of states at the Fermi energy 3 . This was realized for mpdel (1) by assuming a constant density of states with a sharp cutoff at $\pm D$, as the other approaches to scaling do 2 , 3 . There it is assumed that the energy scales of the system do not change as long as the density of states is finite at the Fermi energy, and scales as $\widetilde{\rho}(\epsilon)=\rho(\epsilon / D)$, which means that the band is sufficient structureless to have only one energy scale $D$.

Here this is proved: The first scaling equation (10) can be taken over because of

$$
T \frac{\partial}{\partial T} \rho(\epsilon T / D)=-D \frac{\partial}{\partial D} \rho(\epsilon T / D) .
$$

For the second scaling equation 12 , the term

$$
Y:=V^{2} \int_{-\infty}^{\infty} \rho(\epsilon / D) \Lambda_{2}(\omega+\epsilon) f(\epsilon) d \epsilon
$$

as in Eq. (A2) in Appendix A is examined, which represents an integration over a conduction-electron line. $\Lambda_{2}$ stands for the real part of the $m$ propagators which lie under this conduction-electron line. Differentiating logarithmically with respect to $D$ and substituting $\epsilon:=u D$ yields

$$
D \frac{\partial}{\partial D} Y=(-D) \int_{-\infty}^{\infty} u \rho^{\prime}(u) f(u D) \Lambda_{2}(\omega+u D) d u
$$

The integrand can be neglected outside the interval $-\sqrt{D} \lesssim \omega \lesssim 0$ as in Sec. IIIB. If $\rho$ is sufficiently smooth around the Fermi energy, the integrand contributes significantly only for $-1 \lesssim u \lesssim 1 / \sqrt{D}$. Therefore one can replace $\Lambda_{2}(\omega+u D)$ by its asymptotic value $1 /(-u D)^{m}$, and the Fermi function by its $T=0$ values up to terms of order $1 / D$. $Y$ scales as

$$
D \frac{\partial}{\partial D} Y \propto D^{1-m}
$$

Only the skeleton diagrams of second order $(m=1)$ are relevant:

$$
D \frac{\partial}{\partial D} Y \propto V^{2} \int_{-\infty}^{0} \rho^{\prime}(u) d u=V^{2} \rho(0) .
$$

Thus the second scaling equation is still valid and depends on the density of states only through its value at the Fermi energy.

\section{Magnetic impurity in a superconductor}

If the density of states of the host has still one energy scale $D$, but the density at the Fermi surface vanishes according to a power law, this models magnetic impurities in superconductord 22 with gap nodes:

$$
\begin{aligned}
\rho(\omega) & =(1+r) \bar{\rho}\left(\frac{\omega}{D}\right)^{r}, \\
\int_{-D}^{D} \rho(\omega) d \omega & =2 D \bar{\rho} .
\end{aligned}
$$

This modifies the scaling law (12) accordingly: Every skeleton diagram depends explicitly on $D$ via the integration boundaries, which sorts out the NCA diagrams as the only relevant diagrams. In addition, every skeleton diagram has one factor $D^{-r}$ per loop. Hence, the second scaling equation is

$$
\begin{aligned}
D \frac{\partial}{\partial D} F_{f}= & (1+r)(1-1 / N) \bar{\rho} V^{2} \frac{\partial}{\partial \epsilon_{f}} F_{f} \\
& -r \bar{\rho} V^{2} \frac{\partial}{\partial \bar{\rho} V^{2}} F_{f}-(1+r) \bar{\rho} V^{2} .
\end{aligned}
$$


The first scaling equation remains unchanged. In the limit of large $N$, this scaling equation is consistent with the results of Fradkin23,

$$
T_{K}=D\left(1-\frac{r}{(1+r) J \bar{\rho}}\right)^{1 / r}
$$

\section{ALTERNATIVE DIAGRAMMATIC METHODS}

What conclusions can be drawn from this theory as to the reliability of other diagrammatic approaches, especially their ability to describe the crossover?

\section{A. NCA and the $1 / N$ expansion}

If the system has $N$ internaldegrees of freedom and exhibitclpcal Fermi-liquid behavior, the $1 / N$ expansion becomes exact in the limit of large $N 24$. Contrary to common beliefl2 25 , the $1 / N$ expansion is not suited for the perturbative regime of high temperatures or magnetic fields because it fails to reproduce the diagrammatic renormalization-group results. That is so because the Kondo temperature itself is a function of $1 / N$. Hence it is not possible to describe the crossover within a finite-order $1 / N$ expansion.

The NCA was considered so far as a "self-consistent" $1 / N$ expansion 1425 . However, this does not explain why the NCA values for the static magnetic susceptibility relative to their $T=0$ value agree so well with the respective renormalization-group results26, even for $N=2$. In view of Eq. (17), this now becomes clear.

\section{B. Higher order skeleton diagrams}

One may ask whether it is possible to improve the NCA substantially by incorporating in $\Upsilon$ skeleton diagrams of higher order. However, one has to be aware that then the numerical problems in solving the self-consistency equations become formidable $₫$. To date one has not succeeded to calculate $\chi(0)$, and hence the Wilson number up to order $1 / N^{2}$ by this diagrammatic approach.

\section{Non-self-consistent methods}

Ope way of incorporating higher-order skeleton diagrams in a theory for impurity systems was put forward by Saso 27 in his $T$-matrix approach. The impurity propagators $R_{f}$ were calculated within the NCA. For an observable, these NCA propagators were inserted into the respective skeleton diagrams of orders higher than 2. Such an approach cannot be derived from a variational principal. Hence it will not correctly describe the universal behavior of the impurity.

\section{Coleman's diagram technique}

In Coleman's approach 12 to the Hamiltonian (11), the constraint $n_{f}+n_{b}=1$ is enforced by adding $\lambda\left(n_{f}+n_{b}\right)$ to $H$ and sending $\lambda$ to infinity; consequently, $H_{f}$ in Eq. (1) changes to

$$
H_{f}=\left(\epsilon_{f}+\lambda\right) \sum_{m} f_{m}^{+} f_{m}+\lambda b^{+} b
$$

The scaling equations $(10)$ and (12) are rederived with the help of this diagram technique, and then Coleman's technique is used to discuss the conserving slave-boson approach of Kroha et a 28 .

Now that the unperturbed part $H_{c}+H_{f}$ is a one-particle Hamiltonian, the standard Matsubara-perturbation theory can be developed. The naked propagators from which the diagram technique is built are given by 


$$
\begin{aligned}
R_{m}^{(0)}\left(i \omega_{n}\right) & =1 /\left(i \omega_{n}-\epsilon_{f}-\lambda\right), \\
R_{0}^{(0)}\left(i \omega_{n}\right), & =1 /\left(i \nu_{p}-\lambda\right) \\
R_{c m}^{(0)}\left(i \omega_{n}\right) & =\rho \int_{-D}^{D} \frac{1}{i \omega_{n}-\epsilon} d \epsilon .
\end{aligned}
$$

Here $i \omega_{n}=2 \pi(n+1 / 2) T$ and $i \nu_{p}=2 \pi n T$ are the fermionic and bosonic Matsubara frequencies, respectively. The vertices are displayed in Fig. 1. Again, a dashed line denotes $R_{m}^{(0)}$, a wavy line $R_{0}^{(0)}$, and a solid line represent the propagator of a conduction electron $R_{c m}^{(0)}$. This propagator already contains the sum over all momenta because of the local interaction with the impurity.

Skeleton diagrams can be defined as above as containing no self-energy insertions. The variational principle follows with the help of the functional

$$
\begin{aligned}
& -\frac{\widetilde{\Upsilon}}{T}:=\sum_{m, n, v}\left(3-\frac{2}{v}\right) \Sigma_{m}^{(v)}\left(i \omega_{n}\right) R_{m}\left(i \omega_{n}\right) \\
& +\sum_{m n}\left[\ln \left(i \omega_{n}-\epsilon_{f}-\lambda-\Sigma_{m}\left(i \omega_{n}\right)\right)-\ln \left(i \omega_{n}-\epsilon_{f}-\lambda\right)\right] \\
& +\sum_{m n}\left\{\ln \left(\left[R_{c m}^{(0)}\right]^{-1}-\Sigma_{c m}\left(i \omega_{n}\right)\right)-\ln \left[R_{c m}^{(0)}\right]^{-1}\right\} \\
& -\sum_{p, v}\left[\ln \left(i \nu_{p}-\lambda-\Sigma_{0}\left(i \nu_{p}\right)\right)-\sum_{p} \ln \left(i \nu_{p}-\lambda\right)\right] .
\end{aligned}
$$

The factor $3-2 / v$ arises because every term $\Sigma^{(v)} R$ contains $3 v / 2$ propagators. It is straightforward to show that $\widetilde{\Upsilon}$ is stationary with respect to variations of the propagators iff the Dyson equations hold. By rearranging the internal summation-frequencies one has the following identity:

$$
\begin{aligned}
\sum_{m, n} \Sigma_{m}\left(i \omega_{n}\right) R_{m}\left(i \omega_{n}\right) & =\sum_{m, n} \Sigma_{c m}\left(i \omega_{n}\right) R_{c m}\left(i \omega_{n}\right) \\
& =(-1) \sum_{p} \Sigma_{0}\left(i \nu_{p}\right) R_{0}\left(i \nu_{p}\right),
\end{aligned}
$$

the minus sign in the second line coming from the additional fermion loop 2 .

As in Luttinger's original approachl, one shows that $\widetilde{\Upsilon}$ is equal to the difference of the interacting and noninteracting free energy $F$ :

$$
\widetilde{\Upsilon}=F(V)-F(0)=: \Delta F .
$$

The Hilbert space is a sum of eigenspaces of the number operator $n=\sum_{m} f_{m}^{+} f_{m}+b^{+} b$. The partition function can then be represented as a sum over these subspaces. The subspace $n=0$ describes the noninteracting system. In Coleman's original approach, the physical subspace $n=1$ is projected out by

$$
\lim _{\lambda \rightarrow \infty} \frac{\partial}{\partial e^{-\beta \lambda}} \frac{-\Delta F}{T}=\lim _{\lambda \rightarrow \infty} Z_{f}(\lambda)=Z_{f}
$$

The limit is approached smoothly so that

$$
\lim _{\lambda \rightarrow \infty} \frac{\partial}{\partial \lambda} Z_{f}(\lambda)=0
$$

\section{First scaling equation}

Analogously to Sec. III A, the explicit derivatives with respect to $\rho V^{2}, \epsilon_{f}$, and $\lambda$ are 


$$
\begin{aligned}
\rho V^{2} \frac{\partial}{\partial \rho V^{2}} \frac{\Delta F}{T} & =\sum_{m, n, v}\left[\Sigma_{m}^{(v)}\left(i \omega_{n}\right) R_{m}\left(i \omega_{n}\right)\right], \\
\frac{\partial}{\partial \epsilon_{f}} \frac{\Delta F}{T} & =\sum_{m, n} R_{m}\left(i \omega_{n}\right), \\
\frac{\partial}{\partial \lambda} \frac{\Delta F}{T} & =\sum_{m, n} R_{m}\left(i \omega_{n}\right)-\sum_{p} R_{0}\left(i \nu_{p}\right) .
\end{aligned}
$$

To determine the $T$ dependence of $\widetilde{\Upsilon}$, one has to bear in mind that each Matsubara frequency is proportional to $T$, that each summation over Matsubara frequencies gives a factor $T$, and that in a diagram of order $v$ there are $v / 2$ such summations. Furthermore, $R_{c m}^{(0)}$ depends on $T$ and $D$ only via $D / T$. Together with Eqs. (32), (36), (37), and (38) it follows that

$$
\Delta F=\left(T \frac{\partial}{\partial T}+\epsilon_{f} \frac{\partial}{\partial \epsilon_{f}}+\rho V^{2} \frac{\partial}{\partial \rho V^{2}}+\lambda \frac{\partial}{\partial \lambda}+D \frac{\partial}{\partial D}\right) \Delta F .
$$

By using Eqs. (34) and (35), the analog of the scaling equation (10) follows:

$$
\left(T \frac{\partial}{\partial T}+\epsilon_{f} \frac{\partial}{\partial \epsilon_{f}}+\rho V^{2} \frac{\partial}{\partial \rho V^{2}}+D \frac{\partial}{\partial D}\right) Z_{f}=0
$$

\section{Second scaling equation}

The functional $\widetilde{\Upsilon}$ depends on the cutoff $D$ only via the integration boundaries $\pm D$ in $R_{c m}^{(0)}$. Hence

$$
\begin{aligned}
D \frac{\partial}{\partial D} \frac{\Delta F}{T}= & \sum_{m n}\left[R_{c m}^{(0)}\left(i \omega_{n}\right)\right]^{(-2)}\left(\frac{D}{i \omega_{n}+D}+\frac{D}{i \omega_{n}-D}\right) \\
& \times\left[R_{c m}\left(i \omega_{n}\right)-R_{c m}^{(0)}\left(i \omega_{n}\right)\right] .
\end{aligned}
$$

The propagator difference $R_{c m}-R_{c m}^{(0)}$ can be expanded as

$$
Y:=\left(R_{c m}-R_{c m}^{(0)}\right)\left[R_{c m}^{(0)}\right]^{(-2)}=\Sigma_{c m}+\Sigma_{c m} R_{c m}^{(0)} \Sigma_{c m}+\cdots .
$$

In either case discussed below it will turn out that only the second-order term

$$
\Sigma_{c m}^{(2)}\left(i \omega_{n}\right)=\frac{V^{2} \rho T}{N} \sum_{p} R_{m}\left(i \omega_{n}+i \nu_{p}\right) R_{0}\left(i \nu_{p}\right)
$$

is relevant in the universal limit of large $D$. The propagators $R_{0, m}$ have the spectral decomposition

$$
R_{0, m}(z)=\int \frac{d x}{z-x-\lambda} \rho_{0, m}(x)
$$

and are centered 12 around $\lambda$. After performing Matsubara summations, this gives

$$
\begin{aligned}
D \frac{\partial}{\partial D} \frac{\Delta F^{(2)}}{T}= & \frac{V^{2} \rho}{N} D \beta \sum_{m, \sigma= \pm 1} \iint d x d y \rho_{m}(x) \rho_{0}(y) \\
& \times \frac{[f(x+\lambda)+b(y+\lambda)][f(\sigma D)-f(x-y)]}{\sigma D-x+y},
\end{aligned}
$$

with $f$ and $b$ the Fermi and Bose functions, respectively. The coefficient of $e^{-\beta \lambda}$ in Eq. (44) for large $D$, when combined with Eqs. (37) and (38) yields because of Eq. (34) the desired scaling equation (13).

In Eq. (42) higher-order terms in $\Sigma$ are of higher order in $e^{-\beta \lambda}$, and vanish relatively to $\Sigma_{c m}$ in the limit of large $\lambda$. By the same arguments as given in Sec. IIIB, one can show that skeleton diagrams of higher order in $\Sigma_{c m}$ are irrelevant: Higher-order skeleton diagrams in Eq. (42) contain at least three impurity propagators which have to be evaluated at $i \omega_{n}= \pm D$ to make a contribution $\propto 1 / D^{2}$, or they are of higher order in $e^{-\beta \lambda}$. 


\section{E. Conserving slave-boson approach}

Kroha et al. . skeleton diagrams within Coleman's slave-boson technique. Kroha et al., however, imposed the constraint only in the average $\left\langle n_{f}+n_{b}\right\rangle_{H}=1$. Their method then does not violate the gauge symmetries of the model like the usual slave-boson mean-field approache8. The boundary condition for the free energy is

$$
\frac{\partial}{\partial \lambda} \Delta F=\left\langle n_{f}+n_{b}\right\rangle_{H}=1 .
$$

This includes unphysical states. Consequently, the conduction electrons acquire a self-energy. The first scaling equation (39) now reads

$$
\Delta F=\left(T \frac{\partial}{\partial T}+\epsilon_{f} \frac{\partial}{\partial \epsilon_{f}}+\rho V^{2} \frac{\partial}{\partial \rho V^{2}}+D \frac{\partial}{\partial D}\right) \Delta F+\lambda .
$$

In order to obtain any observable, $\Delta F$ has to be differentiated once more with respect to an external field. Hence this equation is the analog to Eq. (10).

The second scaling equation can be derived from Eq. (44) in the limit of large $D$. First it is summed over $\sigma$. Only the term $\propto f(\sigma D)$ survive because the propagators $R$ have a spectral weight centered around a finite value of $\lambda$, and $\sum_{\sigma} \lim _{D \rightarrow \infty} D /(\sigma D-x+y)=0$. With Eqs. (37), (38), and (45), one has

$$
\begin{aligned}
D \frac{\partial}{\partial D} \frac{\Delta F^{(2)}}{T} & =\frac{-V^{2} \rho \beta}{N} \sum_{m} \iint d x d y \rho_{m}(x) \rho_{0}(y) \\
& \times[f(x+\lambda)+b(y+\lambda)] \\
= & (1-1 / N) V^{2} \rho \frac{\partial}{\partial \epsilon_{f}} \frac{\Delta F^{(2)}}{T}-V^{2} \rho \beta .
\end{aligned}
$$

More generally, because $\sum_{\sigma} \lim _{D \rightarrow \infty} D /\left(i \omega_{n}+\sigma D\right)=0$ in every propagator of Eq. (41) the Matsubara frequency $i \omega_{n}$ can be replaced by $\sigma D$ with an overall factor $f(\sigma D)$. In the magnetic limit one has $\lambda \gtrsim\left|\epsilon_{f}\right|$ to enforce 28 both $n_{f} \approx 1$ and $n_{b} \approx 0$. Therefore higher order terms in Eq. (42) are irrelevant, because they are either $o(1 / D)$, or of higher order in $e^{-\beta \lambda}$.

Hence the approximation also preserves the exact scaling laws. This explains why their results for the $f$ propagator are so similar to the respective NCA results 14 . Moreover, one can now predict that taking into account more skeleton diagrams will not alter the picture qualitatively.

\section{SUMMARY}

The Anderson-impurity model shows, in a nutshell, the difficulties when dealing with strong correlations. One encounters the same problems as in high-energy physics: The perturbation theory of observables at energies $T$ in the coupling constant diverges logarithmically, $\propto \ln D / T$. The limit of zero energy $T$ in solid-state physics corresponds to the limit of infinite cutoff $D$ in high-energy physics. If the Hamiltonian can be shown to be renormalizable, it means that there are finitely many energy scales in the system, at least in the perturbative region. If there is but one scale, the system can be described by a "running" coupling constant $J(D)$, expressed with the help of the $\beta$ function:

$$
\beta(J)=\frac{d J(D)}{d \ln D} .
$$

Reducing the cutoff $D$ of the system does not change its physics as long as the effective coupling $J(D)$ is changed according to Eq. (47), to keep the energy scale constant. However, outside the energy regime where the perturbation theory is valid it has never been shown that such a $\beta$ function exists at all.

In this paper, the variational functional of Luttinger and Ward was used to prove both the existence and form of the $\beta$ function, or, in other words, the exact energy scales of an impurity-system were determined. It turned out that neglecting the vertex corrections (NCA) suffices to proof universal behavior, and that families of higher-order skeleton diagrams do not alter the energy scales. 
The method differs therefore from the conventional renormalization-group approach. Within that approach, the fixed points of a flow of effective Hamiltonians is studied perturbatively as the energy scales of the system are varied. The crossover in inaccessible by that method.

In comparison to that, the skeleton diagrams can describe the crossover very well, as was demonstrated. However, the theory cannot predict the nature of the fixed-point Hamiltonian.

Further work on this subject will concentrate on the question of whether the NCA can also be justified as a means to solve the effective impurity model onto which the infinite-dimensional versions of correlated electron systems can be mapped29. 30 . In particular one would like to learn whether the periodic Anderson Hamiltonian exhibits heavy fermion behavior in this limit, or if this is only an artifact of the approximations used. Also, it should be within the reach of the theory to decide which class of diagrams should be used to describe the problem of two impurities in a metal.

\section{ACKNOWLEDGEMENTS}

It was a pleasure to discuss the intricacies of diagrammatic approaches with Tom Schork and Professor V. Zevin. I would like to thank Professor P. Fulde and G. Zwicknagl for suggesting that I investigate the NCA, and for their constant interest in the progress of this work.

\section{APPENDIX A: IRRELEVANCE OF HIGHER-ORDER SKELETON DIAGRAMS}

The proof uses the spectral decomposition of the ionic propagators $R_{f}$. Every skeleton diagram depends explicitly on $D$ only via the integration boundaries $\pm D$ coming from integration over the conduction-electron lines, as shown in Eq. (6). For $\alpha=0, \ldots, N$, let $\Sigma_{\alpha}^{(2 n)} R_{\alpha}$ be such a skeleton diagram of $2 n$th order with $p$ closed fermion loops and $n$ ionic propagators $R_{i}$ :

$$
\begin{aligned}
& \oint \frac{d z}{2 \pi i} e^{-\beta z} \Sigma_{\alpha}^{(2 n)}(z) R_{\alpha}(z) \\
& =\frac{\rho^{n} V^{2 n}}{N^{n-p}} \oint \frac{d z}{2 \pi i} e^{-\beta z} \prod_{j=1}^{n} \int_{-D}^{D} f\left(\epsilon_{j}\right) d \epsilon_{j} \prod_{i=1}^{n} R_{i}\left(z+\sum_{j}^{(i)} \epsilon_{j}\right)
\end{aligned}
$$

Here $\int d \epsilon_{j}$ denotes the integration over the $j$ th conduction-electron line. Furthermore, the term $\sum_{j}^{(i)}$ in the argument of $R_{i}$ indicates that the sum runs over $j$ if the $i$ th propagator sits under the $j$ th conduction-electron line, as in Fig. 3, the numbers indicating the $\epsilon_{j}$. The $i$ th propagator $R_{i}$ of the respective skeleton diagram has the spectral decomposition

$$
R_{i}\left(z+\sum_{j}^{(i)} \epsilon_{j}\right)=\int_{-\infty}^{\infty} d \lambda_{i} \rho_{i}\left(\lambda_{i}\right) /\left(z+\sum_{j}^{(i)} \epsilon_{j}-\lambda_{i}\right)
$$

For that reason, the line integral gives only contributions from the real poles

$$
z=\omega_{k}=\lambda_{k}-\sum_{j}^{(k)} \epsilon_{j}
$$

For real $\omega$, there holds the relation

$$
\Re R_{0, m}(\omega)=\int_{-\infty}^{\infty} \frac{\rho_{0, m}(\lambda)}{\omega-\lambda} d \lambda,
$$

where the Cauchy-principal value of the integral has to be taken. Hence the $k$ th pole of the line integrals gives

$$
\begin{aligned}
& \prod_{j=1}^{n} \int_{-D}^{D} f\left(\epsilon_{j}\right) d \epsilon_{j} \int_{-\infty}^{\infty} d \omega e^{-\beta\left(\omega-\sum_{j}^{(k)} \epsilon_{j}\right)} \rho_{k}(\omega) \\
& \times \prod_{i \neq k}^{n} \Re R_{i}\left(\omega-\sum_{j}^{(k)} \epsilon_{j}+\sum_{j}^{(i)} \epsilon_{j}\right) .
\end{aligned}
$$


Because of $f\left(\epsilon_{j}\right) e^{\beta \epsilon_{j}}=f\left(-\epsilon_{j}\right)$, one shifts those variables $\epsilon_{j}$ to $-\epsilon_{j}$, which occur in $\sum^{(k)}$ but not in $\sum^{(i)}$ :

$$
\prod_{j=1}^{n} \int_{-D}^{D} f\left(\epsilon_{j}\right) d \epsilon_{j} \int_{-\infty}^{\infty} d \omega e^{-\beta \omega} \rho_{k}(\omega) \prod_{i \neq k}^{n} \Re R_{i}\left(\omega+\sum_{j}^{(i)} \epsilon_{j}\right),
$$

where now $\sum_{j}^{(i)}$ runs over $j$ if the propagator $R_{i}$ sits under the $j$ th conduction-electron line in the respective skeleton diagram with cyclic permutated vertices. In such a diagram, the propagator $R_{k}$ is the outer one.

Exactly here one uses the fact that only whole families of skeleton diagrams are considered: It was just shown that for every family $F$ of $2 n$th order skeleton diagrams their contribution to the line integral in Eq. (5) is

$$
\begin{aligned}
& \operatorname{Tr}_{f} \oint \frac{d z}{2 \pi i} e^{-\beta z} \Sigma_{f}^{(2 n, F)}(z) R_{f}(z)=2 n \int d \omega e^{-\beta \omega} \\
& \times\left[\rho_{0}(\omega) \operatorname{Re} \Sigma_{0}^{(2 n, F)}(\omega)+\sum_{m} \rho_{m}(\omega) \operatorname{Re} \Sigma_{m}^{(2 n, F)}(\omega)\right] .
\end{aligned}
$$

Here, the operator Re is defined to replace every propagator in its argument by its real part. The factor $2 n$ arises because every skeleton diagram occurs in a family $2 n$ times. In $\Upsilon$, every skeleton diagram of order $2 n$ depends explicitly on $D$ only via the integration boundaries $\pm D$ of its $n$ integrations over the conduction-electron lines. The $i$ th integral over a conduction-electron line can be written as

$$
\operatorname{Re} \Sigma_{f}^{(2 n, F)}(\omega)=\left(\int_{-D}^{D}\right)^{n-1} \Lambda_{1}(\omega) \int_{-D}^{D} d \epsilon_{i} f\left(\epsilon_{i}\right) \Lambda_{2}\left(\omega+\epsilon_{i}\right),
$$

where the part $\Lambda_{1}$ of the respective diagram does not depend on $\epsilon_{i}$, that is, its ionic propagators are lying on the left or the right of the $i$ th conduction-electron line. The propagators of part $\Lambda_{2}$ lie under the $i$ th conduction-electron line. $\left(\int\right)^{n-1}$ hints at the other $n-1$ integrations, weighted with the respective Fermi functions. One differentiates with respect to $D$ by evaluating the integral over the $i$ th conduction-electron line $\epsilon_{i}$ at its integration boundaries $\pm D$,

$$
2 n \sum_{\sigma= \pm 1} \int d \omega e^{-\beta \omega} \rho_{0, m}(\omega)\left[\Lambda_{1}(\omega) D f(\sigma D) \Lambda_{2}(\omega+\sigma D)\right]
$$

The integral is to be weighted with $1 / Z_{f}$ in order to yield the $D$ derivative of the respective contribution to the free energy. Because $e^{\beta E_{0}} Z_{f}$ goes for low temperatures to 1 , the spectral densities $\rho_{0, m}$ are weighted with $e^{\beta\left(E_{0}-\omega\right)}$. For low temperatures $T \ll D$, this weighted spectral density contributes to the integral only for frequencies $\omega \lesssim E_{0}$. Because the other integrations over conduction-electron lines $\epsilon_{j}$ are weighted with Fermi functions $f\left(\epsilon_{j}\right)$, respectively, for low temperatures $T \ll D$ the real part of a propagator $\Re R_{f}\left(\omega-D+\sum_{j} \epsilon_{j}\right)$ contributes in $\Lambda_{2}(\omega-D)$ only for frequencies

$$
\omega-D \lesssim E_{0}-D<-D .
$$

In this frequency interval one can replace $\Re R_{f}$ by its naked counterpart $1 /\left(\omega-H_{f}-D\right)$, and hence can estimate its contribution to $F_{f}$ from above as $1 / D$. The term $\propto f(D)$ does not contribute for $T \ll D$. It therefore can be estimated as

$$
\frac{1}{Z} D \frac{\partial}{\partial D} \Sigma_{f}^{(2 n)} R_{f} \propto D / D^{m_{i}} .
$$

Hence at least one $m_{i}$ must equal 1, otherwise the skeleton diagram is irrelevant. However, such a diagram is a skeleton only if it is of second order; otherwise it would have a self-energy insertion. This was shown. Eq. (12) follows.

\section{APPENDIX B: NCA AT ZERO TEMPERATURE}

\section{NCA differential equations}

The NCA equations (6) together-with the Dyson equations (4) constitute a self-consistent system of equations which have been solved numerically 20 . For $T=0$ the NCA integral equations (6) in a magnetic field are transformed 
into differential equations by substituting for the Fermi function a step function 21,16 , 14 , by introducing the negative, inverse of the propagators $R_{0, m}$,

$$
\begin{aligned}
Y_{0}(z) & =\Sigma_{0}^{(2)}(z)-z, \\
Y_{m}(z) & =\Sigma_{m}^{(2)}(z)+\epsilon_{f}-z,
\end{aligned}
$$

and $Y_{m}=\bar{Y}_{m}+m g \mu_{B} h$ for $-j \leq m \leq j=(N-1) / 2$,

$$
\begin{aligned}
\frac{\partial}{\partial \omega} Y_{0} & =-1-\frac{\rho V^{2}}{N} \sum_{m} \frac{1}{\bar{Y}_{m}+m g \mu_{B} h} \\
\frac{\partial}{\partial \omega} \bar{Y}_{m} & =-1-\frac{\rho V^{2}}{N} Y_{0}^{-1} .
\end{aligned}
$$

The inverse propagators have the asymptotic forms

$$
\begin{aligned}
\bar{Y}_{m}(\omega) & \approx-\omega+\epsilon_{f} \\
Y_{0}(\omega) & \approx-\omega
\end{aligned}
$$

for large, negative $\omega$. The NCA differential equations have, up to terms of order $O(1 / D)$ the integral

$$
\begin{aligned}
& \frac{Y_{0}}{\rho V^{2}}+\frac{1}{N} \ln \left|\frac{Y_{0}}{\rho V^{2}}\right| \\
= & \frac{\bar{Y}_{m}}{\rho V^{2}}+\frac{1}{N} \sum_{m} \ln \left|\frac{\bar{Y}_{m}+m g \mu_{B} h}{\rho V^{2}}\right|-\frac{\epsilon_{f}^{*}}{\rho V^{2}} \\
\epsilon_{f}^{*} & =\epsilon_{f}+\left(1-\frac{1}{N}\right) \rho V^{2} \ln \frac{D}{\rho V^{2}} .
\end{aligned}
$$

The value of $\epsilon_{f}^{*}$ follows by inserting Eq. (B3). Terms of order $O(1 / D)$ are neglected because the universal behavior of the system is investigated. The integral of the NCA differential equations contains the energy scales $\rho V^{2}$ and $T_{K}$ via21 $\ln \left(T_{K} / \rho V^{2}\right)=\epsilon_{f}^{*} / \rho V^{2}$. It is a nontrivial task to solve this differential equations numerically in the universal, magnetic limit

$$
\begin{aligned}
D & \rightarrow \infty, \quad \epsilon_{f} \rightarrow-\infty \\
\lim _{D \rightarrow \infty} \frac{\epsilon_{f}}{D} & =0, \quad \frac{V^{2}}{\left|\epsilon_{f}\right|}=J=\text { const.. }
\end{aligned}
$$

\section{What is the ground-state energy of the NCA?}

The ground-state energy of the NCA is defined as

$$
E_{0}^{\mathrm{NCA}}=\lim _{T \rightarrow 0} F_{f}^{\mathrm{NCA}} .
$$

However, one has not yet succeeded in deriving an expression for $E_{0}^{\mathrm{NCA}}$ via that route, but merely solves the NCAdifferential equations ( $(\overline{B 2})$. It has been conjectured 14 that the lowest, real zeros of the inverse propagators $Y_{0, m}$ define the NCA ground-state energy. This is now proved: Because of Eq. (B5) $E_{0}^{\mathrm{NCA}}$ fulfills

$$
1=\lim _{T \rightarrow 0} e^{\beta E_{0}^{\mathrm{NCA}}} \int e^{-\beta \omega}\left[\rho_{0}^{\mathrm{NCA}}(\omega)+\sum_{m} \rho_{m}^{\mathrm{NCA}}(\omega)\right] d \omega .
$$

Hence, for $T=0$ there exist the (positive) spectral densities

$$
\widehat{\rho}_{0, m}^{\mathrm{NCA}}(\omega)=e^{\beta\left(E_{0}^{\mathrm{NCA}}-\omega\right)} \rho_{0, m}^{\mathrm{NCA}}(\omega),
$$


and therefore the spectral densities $\rho_{0, m}^{\mathrm{NCA}}$ vanish for $T=0$ and $\omega<E_{0}^{\mathrm{NCA}}$. This means that the inverse propagators $Y_{0, m}$ are real for those frequencies. In addition, $\widehat{\rho}_{0, m}^{\mathrm{NCA}}$ vanish for $\omega>E_{0}$, because $\rho_{0, m}^{\mathrm{NCA}}$ remain finite. However, from the existence theorem for solutions of the differential equations (B2) it follows that there exists at least one value $\omega_{0}$ such that the following hold.

(1) $Y_{0, m}$ have a zero in $\omega_{0}$ and become complex above, if they are real for large, negative $\omega$ - which they are in view of Eq. (B3). Because it was just shown that the $Y_{0, m}$ are real for $\omega<E_{0}^{\mathrm{NCA}}$, they are real for $\omega<\omega_{0}$ as well.

(2) $\rho_{0, m}^{\mathrm{NCA}}$ vanish for $\omega<\omega_{0}$, therefore $\omega_{0} \leq E_{0}^{\mathrm{NCA}}$.

(3) $\widehat{\rho}_{0, m}^{\mathrm{NCA}}$ vanish for $\omega<\omega_{0}$, therefore $\omega_{0} \geq E_{0}^{\mathrm{NCA}}$.

To sum up: $\omega_{0}$ is the lowest common zero for $Y_{0, m}$, and at the same time the NCA ground-state energy.

\section{Parametrization of the NCA ground-state energy}

Hence the well-known expression for the common zero of $Y_{0 . m} 21,261,22$ can be used as the NCA ground-state energy and is parametrized in the following manner: Define the $W$-function for positive $x$ as

$$
W(x) \exp [W(x)]=x \quad \text { for } x \geq 0,
$$

with asymptotic behaviors

$$
\begin{aligned}
W(x) & =x+o\left(x^{2}\right) \quad \text { for } x \rightarrow 0^{+}, \\
W[\exp (x)] & =x-\ln (x)+o(1) \quad \text { for } x \rightarrow \infty .
\end{aligned}
$$

The integral (B4) can be solved for $Y_{0}$ because both $Y_{0}$ and $Y_{m}$ are positive for $\omega<E_{0}^{\mathrm{NCA}}$ :

$$
\begin{aligned}
& N V^{-2} Y_{0} \\
& =W\left(N \exp \left[\left(\bar{Y}_{m}-\epsilon_{f}^{*}\right) \frac{N}{\rho V^{2}}+\sum_{m} \ln \frac{\bar{Y}_{m}+m g \mu_{B} h}{\rho V^{2}}\right]\right) .
\end{aligned}
$$

$E_{0}^{\mathrm{NCA}}$ can now be parametrized with the help of

$$
E_{0}=D+\int_{-D}^{E_{0}} d \omega=D+\int_{\bar{Y}_{m}(-D)}^{j g \mu_{B} h} \frac{d \omega}{d \bar{Y}_{m}} d \bar{Y}_{m}
$$

the NCA differential equations (B2), and Eq. (B9) as

$$
\begin{aligned}
& E_{0}=\epsilon_{f}-j g \mu_{B} h-\int_{j g \mu_{B} h}^{D+\epsilon_{f}} d \omega \\
& \times \frac{1}{1+W\left(N \exp \left[\left(\omega-\epsilon_{f}^{*}\right) \frac{N}{\rho V^{2}}+\sum_{m} \ln \frac{\omega+m g \mu_{B} h}{\rho V^{2}}\right]\right)} .
\end{aligned}
$$

For vanishing magnetic field there exists another parametrization because $Y_{m}$ can be written as a function of $Y_{0}$ with the help of the $W$ function:

$$
E_{0}=-\int_{0}^{D} \frac{d \omega}{1+W\left(\exp \left[\left(\omega+\epsilon_{f}^{*}\right) \frac{1}{\rho V^{2}}+\frac{1}{N} \ln \frac{\omega}{\rho V^{2}}\right]\right)} .
$$

The NCA ground-state energy fulfills the equations (10) and (12), as can be checked with the help of (B11). The ground-state energy is not an universal fungtion because of the constant term $-\rho V^{2}$ in Eq. (12). The ground-state energy up to order $1 / N$ and $1 / D$ follows as 2 :

$$
\begin{aligned}
E_{0} & =\epsilon_{f}+\rho V^{2} W\left[\frac{D}{\rho V^{2}} \exp \left(\frac{\epsilon_{f}}{\rho V^{2}}\right)\right] \\
& +\frac{1}{N} \int_{0}^{D} \frac{W(y)}{[1+W(y)]^{3}} \ln \frac{x}{D} d x+O(1 / D)+O\left(1 / N^{2}\right) \\
y & =\frac{D}{\rho V^{2}} \exp \left(\frac{x+\epsilon_{f}}{\rho V^{2}}\right) .
\end{aligned}
$$




\section{An analytical expression for the static magnetic susceptibility of the NCA}

\section{a. Universality}

With the help of the Kondo temperature (14), Eq. (B10) reads, in the magnetic limit $T_{K} / \rho V^{2} \rightarrow 0$ after the substitution $\omega / T_{K}=x$,

$$
\begin{aligned}
E_{0}= & \epsilon_{f}-j g \mu_{B} h-T_{K} \int_{j g \mu_{B} h / T_{K}}^{\left(D+\epsilon_{f}\right) / T_{K}} d x \\
& \times \frac{1}{1+W\left\{N \exp \left[\sum_{m} \ln \left(x+\frac{m g \mu_{B} h}{T_{K}}\right)\right]\right\}} .
\end{aligned}
$$

After differentiating with respect to the magnetic field $h$ the integrand decays rapidly enough so that one can replace the upper limit of integration by $\infty$. Hence the magnetization scales as a function of $h$ as

$$
M\left(h, D, \epsilon_{f}, V, N\right)=M\left(\frac{g \mu_{B} h}{T_{K}}\right) .
$$

This shows explicitly that already the skeleton diagrams of second order give the exact energy scale for the spin degrees of freedom.

\section{b. Small magnetic fields}

Using the parametrization (B10), the magnetization $M(h)=-\partial_{h} E_{0}$ vanishes for $h=0$ begause of $\sum_{m} m=0$. The second derivative gives the static magnetic susceptibility for $h=0$. With the abbreviation 14

$$
\frac{1}{3} \mu_{j}^{2} N=\left(g \mu_{B}\right)^{2} \sum_{m} m^{2},
$$

from Eq. (B10) one has, after substituting $N Y_{0} / \rho V^{2}=W$, using the NCA differential equations, and finally partially integrating,

$$
\begin{aligned}
\chi(0) & =\frac{1}{3} \mu_{j}^{2} \frac{1}{\rho^{2} V^{4}} \int_{0}^{D} \frac{2 W(y)+1}{W(y)[1+W(y)]^{3}} d x \\
y & =\left(\frac{x}{\rho V^{2}}\right)^{\frac{1}{N}} \frac{T_{K}}{\rho V^{2}} \exp \left(\frac{x}{\rho V^{2}}\right) .
\end{aligned}
$$

In the magnetic limit, $y$ is very small for $x<\left|\epsilon_{f}^{*}\right|$ because of $-\epsilon_{f}^{*} \gg \rho V^{2}$, and above that $W>1$ and the integrand is small. Therefore one can replace the $W$ function by its argument, and extend the integration to $\infty$ :

$$
\begin{aligned}
\chi^{\mathrm{NCA}}(0) & =\frac{1}{3} \mu_{j}^{2} \frac{1}{T_{K}} \int_{0}^{\infty} e^{-t} t^{-1 / N} d t \\
& =\frac{1}{3} \mu_{j}^{2} \frac{1}{T_{K}} \Gamma(1-1 / N) .
\end{aligned}
$$

The exact result

$$
\chi(0)=\frac{1}{3} \mu_{j}^{2} \frac{1}{T_{K}} \frac{1}{\Gamma(1+1 / N)}
$$

was obtained by fitting the results of the Bethe ansatz to perturbation theory in the nonmagnetic limit $\mathrm{E}$. Both results coincide up to order $1 \mathrm{LN}$, because the NCA contains all diagrams up to that order. This contradicts the claim of Kuramoto and Kojima 32 that the NCA would yield the exact result for $\chi(0)$ in the magnetic limit. 


\section{APPENDIX C: COQBLIN-SCHRIEFFER MODEL}

\section{Variational functional}

The Schrieffer-Wolff transformation 14 projects - up to a constant - the Anderson model onto the Coqblin-Schrieffer model in the magnetic limit $\epsilon_{f} \rightarrow-\infty$ and constant $J=V^{2} /\left|\epsilon_{f}\right|$,

$$
\begin{aligned}
H & \mapsto H_{\mathrm{CS}}-2 \frac{\rho J D}{N} \\
H_{\mathrm{CS}} & =\sum_{\left|\epsilon_{p}\right|<D, m} \epsilon_{p} c_{p m}^{+} c_{p m}+\frac{J}{N} \sum_{p q, m n} c_{p m}^{+} c_{q n} f_{n}^{+} f_{m} .
\end{aligned}
$$

For that the spectrum of $H$ is shifted at $\left(-\epsilon_{f}\right)$ by shifting the argument $z$ in $\Upsilon$ at $\epsilon_{f}$, and the propagators and self-energies are transformed ast 4

$$
\begin{aligned}
\frac{1}{\epsilon_{f}} \Sigma_{0}\left(z+\epsilon_{f}\right) & \rightarrow \Pi(z), \\
\frac{1}{1+\frac{z}{\epsilon_{f}}-\frac{1}{\epsilon_{f}} \Sigma_{0}\left(z+\epsilon_{f}\right)} & \rightarrow R_{0}(z)=\frac{1}{1-\Pi(z)} \\
R_{m}\left(z+\epsilon_{f}\right) & \rightarrow R_{m}(z)=\frac{1}{z-\Sigma_{m}(z)} .
\end{aligned}
$$

A $2 n$th order diagram carries the prefactor $(-1)^{n}(\rho J)^{n}$. Because $\left|\epsilon_{f}\right| \gg D,|z|$, the term $z / \epsilon_{f}$ was neglected in $\epsilon_{f} R_{0}\left(z+\epsilon_{f}\right)$ and such the charge degrees of freedom of the impurity are projected out. The variational functional $\Upsilon$ now reads

$$
\begin{aligned}
\Upsilon= & \beta \oint \frac{d z}{2 \pi i} e^{-\beta z}\left\{\sum_{m, n}\left(1-\frac{1}{n}\right) \Sigma_{m}^{(n)}(z) R_{m}(z)\right. \\
& +\sum_{n}\left(1-\frac{1}{n}\right) \Pi^{(n)}(z) R_{0}(z) \\
& \left.+\ln \left[z-\sum_{n} \Sigma_{m}^{(n)}(z)\right]+\ln \left[1-\sum_{n} \Pi^{(n)}(z)\right]\right\}
\end{aligned}
$$

The saddle-point property of $\Upsilon$ is shown as Bickers by 14 .

\section{Skeleton diagrams of second order}

The analog to the NCA is called the "self-consistent ladder approximation" 4 . The NCA equations follow from Eq. (6) after projecting as in the last paragraph 14 .

$$
\begin{aligned}
\Sigma_{m}(z) & =-\frac{\rho J}{N} \int_{-D}^{D} f(\epsilon) \frac{1}{1-\Pi(z+\epsilon)} d \epsilon \\
\Pi(z) & =-\frac{\rho J}{N} \sum_{m} \int_{-D}^{D} f(\epsilon) R_{m}(z+\epsilon) d \epsilon .
\end{aligned}
$$

The first scaling equation follows as in Sec. III A:

$$
F_{f}=T \frac{\partial}{\partial T} F_{f}+D \frac{\partial}{\partial D} F_{f}
$$

The asymptotic behavior of the self-energies is estimated from Eq. (C3) for $|\omega| \ll D$ as

$$
\begin{aligned}
\Sigma_{m}(\omega-D) & =-\rho J D / N+O\left(J^{2}\right) \\
\Pi(\omega-D) & =O(J \ln D) .
\end{aligned}
$$


Because of Eq. (C1), $z$ has to be shifted in every propagator by $-2 \rho J D / N$, to describe the Coqblin-Schrieffer model. Therefore

$$
\begin{aligned}
R_{m}(\omega-D) & =\frac{1}{1+\rho J / N} \frac{-1}{D} \text { for }|\omega| \ll D, \\
R_{0}(\omega-D) & =1 \text { for }|\omega| \ll D .
\end{aligned}
$$

Higher orders in $J$ are irrelevant as will be shown below. It follows within the NCA that

$$
D \frac{\partial}{\partial D} Z_{f}=-\beta J \oint \frac{d z}{2 \pi i} e^{-\beta z}\left(\frac{R_{0}(z)}{1+\rho J / N}-D R_{m}(z)\right) .
$$

Hence the scaling equation for the impurity part of the free energy is

$$
D \frac{\partial}{\partial D} F_{f}^{\mathrm{NCA}}=\frac{(\rho J)^{2}}{1+\rho J / N} \frac{\partial}{\partial \rho J} F_{f}^{\mathrm{NCA}}-\frac{\rho J}{N} D .
$$

This equation does not change in the universal limit $J \ll D$ if higher orders of $J$ in Eq. (C6) are taken into account. The following scaling law holds therefore for the impurity part of the free energy:

$$
\left(F_{f}-E_{0}\right)^{\mathrm{NCA}}(T, D, J)=\left(F_{f}-E_{0}\right)^{\mathrm{NCA}}\left(\frac{T}{T_{K}^{\mathrm{CS}}}\right)
$$

with the Kondo temperature of the Coqblin-Schrieffer model?

$$
T_{K}^{\mathrm{CS}}=D \sqrt[N]{\rho J} \exp [-1 / \rho J] .
$$

For other observables, one has to couple $H$ to suitable external fields. In particular, the Kondo resonance is reproduced qualitatively correctly.

\section{Skeleton diagrams of higher order}

The two limits $D \rightarrow \infty$ and $\epsilon_{f} \rightarrow-\infty$ are not interchangeable, as can be seen from comparing the Kondo temperatures of Eqs. (14) and (C9). Skeleton diagrams of higher order than two are relevant in $\Upsilon$. The reason for that is the asymptotic behavior of $R_{0}$ which goes to one at the cutoff. If the diagram in Fig. 3 is logarithmically differentiated with respect to $D$, the contribution of the second conduction-electron line does not vanish for large $D$. In particular it is not possible to prove now that the energy scale (C9) is the exact one by considering only skeleton diagrams of second order. In fact, the NCA still predicts for $N=1$ a low-energy scale ( $\overline{\mathrm{C} 9})$ although there is none.

\section{NCA at zero temperature}

The derivations are as in Appendix B. The inverse of the pseudopropagator $\Pi$ is defined to be $Y_{0}=1-\Pi$. The NCA differential equations are up to terms $\propto 1 / D$,

$$
\begin{aligned}
\frac{\partial}{\partial \omega} Y_{0} & =\frac{-\rho J}{N} \sum_{m} Y_{m}^{-1}(\omega), \\
\frac{\partial}{\partial \omega} Y_{m} & =-(1-\rho J / N)-\frac{\rho J}{N} Y_{0}^{-1}(\omega) .
\end{aligned}
$$

With $Y_{m}=\bar{Y}_{m}+m g \mu_{B} h$ and $\widetilde{D}=D(1-\rho J D / N)$ the NCA differential equations have up to terms of order $1 / D$ the integral

$$
\begin{aligned}
& Y_{0}\left(\frac{1}{\rho J}-\frac{1}{N}\right)+\frac{1}{N} \ln Y_{0} \\
& =\frac{1}{N} \sum_{m} \ln \left|\frac{\bar{Y}_{m}+m g \mu_{B} h}{\widetilde{D}}\right|+\left(\frac{1}{\rho J}-\frac{1}{N}\right) .
\end{aligned}
$$


The ground-state energy is expressible as

$$
E_{0}=-A+\int_{-A}^{E_{0}} d \omega
$$

where $A$ is a still arbitrary constant. If $J \ll A \ll D$ and $\lim _{D \rightarrow \infty} A=\infty$, the integral (C11) can be used as in Appendix B to yield, in the universal limit of small $\rho J$,

$$
\chi^{\mathrm{NCA}}(0)=\frac{1}{3} \mu_{j}^{2} \frac{1}{T_{K}^{\mathrm{CS}}} \frac{\Gamma(1-1 / N)}{\sqrt[N]{e}} .
$$

which is up to $O\left(1 / N^{2}\right)$ identicall to the result of Rasul and Hewson, where $\Gamma(1-1 / N)$ is replaced by $1 / \Gamma(1+1 / N)$.

${ }^{1}$ G. Grüner and A. Zawadowski, Rep. Prog. Phys. 37, 1497 (1974).

2 A. C. Hewson, The Kondo Problem to Heavy Fermions (Cambridge University Press, Cambridge, 1993).

${ }^{3}$ N. Andrei, K. Furuya, and J. H. Lowenstein, Rev. Mod. Phys. 55, 331 (1983).

${ }^{4}$ P. B. Wiegmann and A. M. Tsvelick, Adv. Phys. 32, 453 (1983).

${ }^{5}$ J. W. Rasul and A. C. Hewson, J. Phys. C 17, 3337 (1984).

${ }^{6}$ K. G. Wilson, Rev. Mod. Phys. 47, 773 (1975).

${ }^{7}$ H. Keiter and J. C. Kimball, Phys. Rev. Lett. 25, 672 (1970).

${ }^{8}$ A. A. Abrikosov and A. A. Migdal, J. Low Temp. Phys. 3, 519 (1970).

${ }^{9}$ J. M. Luttinger and J. C. Ward, Phys. Rev. 118, 1417 (1960).

${ }^{10}$ Y. Kuramoto, Z. Phys. B 53, 37 (1983).

${ }^{11}$ G. Baym, Phys. Rev. 127, 1391 (1962).

${ }^{12}$ P. Coleman, Phys. Rev. B 29, 3035 (1984).

${ }^{13}$ P. Fulde, Electron Correlations in Molecules and Solids (Springer-Verlag, Berlin, 1995).

${ }^{14}$ N. Bickers, Rev. Mod. Phys. 59, 845 (1987).

15 N. Grewe, Z. Phys. B 53, 271 (1983).

${ }^{16}$ Y. Kuramoto, Z. Phys. B 57, 95 (1984).

${ }^{17}$ F. D. M. Haldane, Phys. Rev. Lett. 40, 416 (1978).

${ }^{18}$ F. B. Anders, J. Phys. Condens. Matter 7, 2801 (1995).

${ }^{19}$ H. Keiter and G. Morandi, Phys. Rep. 109, 227 (1984).

${ }^{20}$ N. E. Bickers, D. L. Cox, and J. W. Wilkins, Phys. Rev. B 36, 2036 (1987).

${ }^{21}$ E. Müller-Hartmann, Z. Phys. B 57, 281 (1984).

${ }^{22}$ K. Satori, H. Shiba, O. Sakai, and Y. Shimizu, J. Phys. Soc. Jpn. 61, 3239 (1992).

${ }^{23}$ D. Withoff and E. Fradkin, Phys. Rev. Lett. 64, 1835 (1990).

${ }^{24}$ N. Read and D. M. Newns, J. Phys. C 17, 3273 (1983).

${ }^{25}$ Y. Kuroda and B. Jin, J. Phys. Soc. Jpn. 57, 1687 (1988).

${ }^{26}$ F. C. Zhang and T. K. Lee, Phys. Rev. B 30, 1556 (1984).

27 T. Saso, J.Magn.Magn.Mat. 76\&77, 176 (1988).

${ }^{28}$ J. Kroha, P. J. Hirschfeld, K. A. Muttalib, and P. Wölfle, Solid State Commun. 83, 1003 (1992).

${ }^{29}$ W. Metzner and D. Vollhardt, Phys. Rev. Lett. 62, 324 (1989).

${ }^{30}$ E. Müller-Hartmann, Z. Phys. B 74, 507 (1989).

${ }^{31}$ S. Inagaki, Prog. Theor. Phys. 62, 1441 (1979).

${ }^{32}$ Y. Kuramoto and H. Kojima, J. Magn. Magn. Mater. 47\&48, 329 (1985).

FIG. 1. Vertices for the Anderson model. A dashed line represents the naked propagator of the occupied $f^{1}$ configuration with internal quantum number $m$. A wavy line represents the naked propagator of the unoccupied $f^{0}$ configuration. A solid line represents the propagator of a conduction electron with internal quantum number $m$. Every diagram has a spine of alternating wavy and dashed impurity propagators. The conduction-electron propagators carry no self-energy 14.

FIG. 2. Skeleton diagram for $\Sigma_{0}^{(2)}\left(R_{m}(z)\right) R_{0}(z)$. The last vertex can be identified with the first because of the trace over the $f$ configurations in Eq. (5). The double, dashed and double, wavy lines represent dressed propagators. 
FIG. 3. Skeleton diagram of order 6 for $\Sigma_{0} R_{0}$ 